\title{
Enquête ethnobotanique sur les plantes utilisées dans le traitement de l'infertilité masculine au Benin
}

\author{
S. AZONBAKIN ${ }^{1 *}$, P. DANGBEMEY ${ }^{2}$, R. OSSENI ${ }^{1}$, S.A. YAUDE ${ }^{1}$, F. KORA $^{1}$,

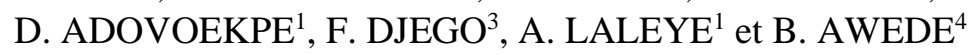 \\ ${ }^{1}$ Laboratoire d'Histologie Biologie de la Reproduction Cytogénétique et Génétique Médicale, Faculté des \\ Sciences de la Santé-Université d'Abomey-Calavi, Benin. \\ ${ }^{2}$ Service de Gynécologie-Obstétrique-Centre Hospitalier et Universitaire de la Mère et de l'Enfant (CHU- \\ $M E L)$, Benin. \\ ${ }^{3}$ Laboratoire d'Ecologie Appliquée, Faculté des Sciences Agronomiques, Université d'Abomey-Calavi, Benin. \\ ${ }^{4}$ Unité de Physiologie Humaine, Faculté des Sciences de la Santé-Université d'Abomey-Calavi, Benin. \\ *Auteur correspondant ; E-mail : azandeg@yahoo.fr, Tel :00229 97130061
}

\begin{tabular}{lll}
\hline Received: 11-01-2021 & Accepted: 22-08-2021 & Published: 31-08-2021 \\
\hline
\end{tabular}

\section{RESUME}

L'infertilité conjugale concerne environ $15 \%$ de la population en Afrique subsaharienne. C'est une affection ayant une implication socioculturelle importante puisqu'au Bénin, l'un des objectifs du mariage est la procréation. L'objectif du présent travail est de contribuer à l'étude des principales plantes médicinales utilisées en médecine traditionnelle pour le traitement de l'infertilité masculine dans les départements de l'Atacora et de l'Atlantique au Bénin. Elle s'est déroulée auprès de 80 praticiens dont 60 tradithérapeutes, 11 personnes ressources et 9 vendeuses de plantes. L'étude a permis de recenser 109 espèces de plantes. Ces espèces appartiennent à 101 genres et sont réparties en 54 familles. Les feuilles sont majoritairement utilisées $(35,77 \%)$ suivies des racines $(29,93 \%)$ tandis que les autres parties (fruit, écorce, graines, plante entière, tige feuillée...) sont moins utilisées dans la préparation des recettes. La décoction est le mode de préparation majoritairement utilisée soit $33,55 \%$ suivie de la macération $27,63 \%$ et de la poudre $25,66 \%$. La trituration et l'infusion sont moins employées à des fréquences respectives de $11,84 \%$ et $1,32 \%$. Ces résultats ont permis de montrer la richesse de la flore béninoise en ressources pour la prise en charge de l'infertilité masculine.

(C) 2021 International Formulae Group. All rights reserved.

Mots clés : Infertilité masculine, plantes médicinales, Atlantique, Atacora

\section{Ethnobotanical survey of plants used in the treatment of male infertility in Benin}

\begin{abstract}
Couple infertility affects around $15 \%$ of the population in sub-Saharan Africa. It is a condition with an important socio-cultural implication because in Benin, one of the objectives of marriage is procreation. The objective of this work is to contribute to the study of the main medicinal plants used in traditional medicine for the treatment of male infertility in the departments of Atacora and Atlantic in Benin. It took place with 80 practitioners including 60 traditional therapists, 11 resource people and 9 plant sellers. The study identified 109 species of plants. These species belong to 101 kinds and are divided into 54 families. The leaves are mostly used
\end{abstract}


(35.77\%) followed by the roots $(29.93 \%)$ while the other parts (fruit, bark, seeds, whole plant, leafy stalk, etc.) are used less in the preparation of recipes. The decoction is the method of preparation mainly used, ie $33.55 \%$ followed by maceration $27.63 \%$ and powder $25.66 \%$ Trituration and infusion are used less at frequencies of $11.84 \%$ and $1.32 \%$, respectively. These results made it possible to show the richness of the Beninese flora in resources for the management of male infertility.

(C) 2021 International Formulae Group. All rights reserved.

Keywords: Male infertility, medicinal plant, Atacora, Atlantic.

\section{INTRODUCTION}

L'infertilité du couple est définie par l'Organisation Mondiale de la Santé (OMS) comme étant l'absence de grossesse chez un couple en âge de procréer au bout de douze mois de rapports sexuels réguliers sans contraception (OMS, 2010). Elle est de nos jours, un réel problème de santé publique. L'infertilité conjugale concerne $10 \%$ des couples dans le monde, soit 60 à 80 millions d'hommes et de femmes. Un couple sur dix est confronté à une infertilité primaire ou secondaire (Sifroi, 2000 ; Brzakowski, 2009). En Afrique subsaharienne, l'infertilité touche 25 à $40 \%$ de la population (Jimoh, 2012). Il y a quelque temps, la femme était considérée comme la seule responsable de l'infertilité du couple. Mais de nos jours, l'on se rend compte que les deux sexes sont touchés avec une part importante de l'infertilité masculine, allant jusqu'à $30 \%$ de causes masculines (Agarwal, 2015). De nombreux médicaments utilisés actuellement, dérivent de plantes qui produisent des métabolites pouvant traiter l'infertilité masculine devenue également un problème de santé publique. Ces traitements proposés par la médecine moderne sont souvent trop coûteux, inaccessibles, d'efficacité discutable et possèdent parfois de nombreux effets secondaires (Zohoun, 1997 ; Byg, 2001). De plus, les structures hospitalières capables d'offrir de tels traitements sont généralement très éloignées des zones rurales (Monteiro, 2006; Jimoh, 2012 ; Akour, 2015). Les populations rurales se tournent alors vers les plantes médicinales comme ultime voie de recours pour résoudre ce problème d'infertilité.

Malheureusement dans notre pays, peu d'études se sont intéressées au traitement de l'infertilité par les plantes. Elles sont parfois utilisées sans aucune preuve scientifique de leur efficacité et de leur innocuité (Reddy, 2009 ; Djego, 2011). L'objectif de ce travail était de contribuer à l'étude du niveau de connaissances et d'utilisation des phytothérapeutes, vendeurs de plantes et personnes-ressources résidant dans les départements de l'Atacora et de l'Atlantique, relatives aux plantes utilisées dans le traitement de l'infertilité masculine.

\section{MATERIEL ET METHODES \\ Cadre d'étude}

Notre étude s'est déroulée dans les départements de l'Atlantique et de l'Atacora où nous avons effectué un recensement des recettes utilisées dans le traitement de l'infertilité masculine au cours des mois de Juillet à Septembre de l'année 2018. Ces deux départements sont situés dans le nord et le Sud du Bénin (Figure 1). Elle a été suivie d'une phase de sélection des recettes significatives au Laboratoire d'Ecologie Appliquée (LEA) de la Faculté des Sciences Agronomiques (FSA) de l'Université d'Abomey-Calavi (UAC) et qui ont fait l'objet de l'expérimentation chez l'homme.

\section{Matériel}

A l'issue de l'inventaire des plantes utilisées dans le traitement traditionnel de l'infertilité masculine, celles sélectionnées ont été récoltées sur place puis identifiées au Laboratoire d'Ecologie Appliquée (LEA/FSA/UAC).

\section{Méthodes}

\section{Type et période d'étude}

L'étude est de type descriptif, analytique et transversal. Elle s'est effectuée du $1^{\text {er }}$ Juillet au 30 Septembre 2018. 


\section{Population d'étude}

Elle est constituée par les vendeurs de plantes, les phytothérapeutes et des personnes ressources résidant dans la zone d'étude.

\section{Critères d'inclusion}

- Être phytothérapeute, vendeur ou vendeuse de plantes médicinales ou personnes ressources (ayant une bonne connaissance des plantes médicinales et de leurs usages)

- Avoir donné son consentement pour l'étude

- Résider dans la zone d'étude et être connu par la population

\section{Critères de non inclusion}

- Refus de participer à l'étude

- Utilisation d'autre chose que la plante dans le traitement de l'infertilité

\section{Echantillonnage}

La technique d'échantillonnage non probabiliste a été utilisée. Grâce à un répertoire de phytothérapeutes résidant par département, un échantillonnage de convenance a été effectué afin d'identifier parmi eux, ceux qui étaient disponibles. Ainsi notre premier échantillon a été sélectionné, puis, au cours du sondage, un échantillonnage par «boule de neige » a été réalisé. En effet, à chaque entrevue avec un enquêté, celui-ci identifiait à son tour un ou plusieurs autres praticiens vers qui nous étions orientés.

\section{Collecte des données}

Les données ont été collectées par la méthode d'interview standardisée. Nous avons utilisé la technique d'entretien individuel et de groupe. A cet effet, deux questionnaires ont été élaborés : l'un destiné aux vendeuses de plantes médicinales (herboristes) et l'autre aux phytothérapeutes.

L'échantillon de chaque plante citée a été collecté en présence des enquêtés et conservé sous forme d'herbier. Ces échantillons ont ensuite été convoyés au Laboratoire d'Ecologie Appliquée de la Faculté des Sciences Agronomiques où leur identification a été faite.

\section{Traitement de données ethnobotaniques}

Pour connaître le niveau de connaissance et d'utilisation des espèces et leur répartition à travers les enquêtes, une analyse quantitative basée sur le calcul des indices a été faite.

\section{$>$ Identification et sélection des recettes significatives}

Selon la méthode de Tramyl IV, une plante ou une recette a été retenue comme significative pour traiter l'infertilité masculine, si plus de $20 \%$ des enquêtés l'ont indiquée. Si aucune des plantes n'a répondu à ce critère, les plantes ou recettes les plus fréquemment citées ont été retenues selon les calculs des indices suivants (Djego, 2011).

- La valeur de diversité (ID) de l'enquêté

C'est le nombre de fois qu'une utilisation a été citée par l'enquêté (Ux) divisé par le nombre total d'utilisation (Ut). Elle est donnée par :

\section{ID $=\mathbf{U x} / \mathbf{U t}$}

- La valeur d'équitabilité (IE) de l'enquêté C'est la valeur de diversité (ID) divisée par l'indice maximal de valeur de diversité obtenue. Elle est donnée par : IE $=$ ID/IDmax

- La valeur consensuelle pour des types d'utilisation (CTU) (Monteiro, 2006)

C'est le nombre de fois qu'une utilisation a été citée (TU) divisé par le nombre total d'utilisations (Ut). Cette valeur a été ensuite divisée par les types d'utilisation regroupés suivant chaque catégorie. Elle a été donnée par : $\mathbf{C T U}=\mathbf{T U} / \mathbf{U t}$

\section{- La valeur de diversité d'utilisation (ID)}

C'est le nombre d'utilisations par catégorie (alimentaire, médicinal ; etc) (Ucx) divisé par le nombre total d'utilisations pour toutes les catégories (Uct). Elle est donnée par :

\section{$\mathrm{UD}=\mathrm{Ucx} / \mathrm{Uct}$}

Une analyse comparative basée sur ces différents indices est réalisée pour évaluer les connaissances des enquêtés et leur niveau d'utilisation des espèces utilisées pour traiter l'infertilité chez l'homme, selon la catégorie socioprofessionnelle, l'âge et le sexe. Ainsi, tous les enquêtés seront regroupés en groupes socioprofessionnels (phytothérapeutes, herboristes, personnes ressources) d'une part et suivant le sexe d'autre part (Rwangabo, 1993 ; Zohoun, 1997). 


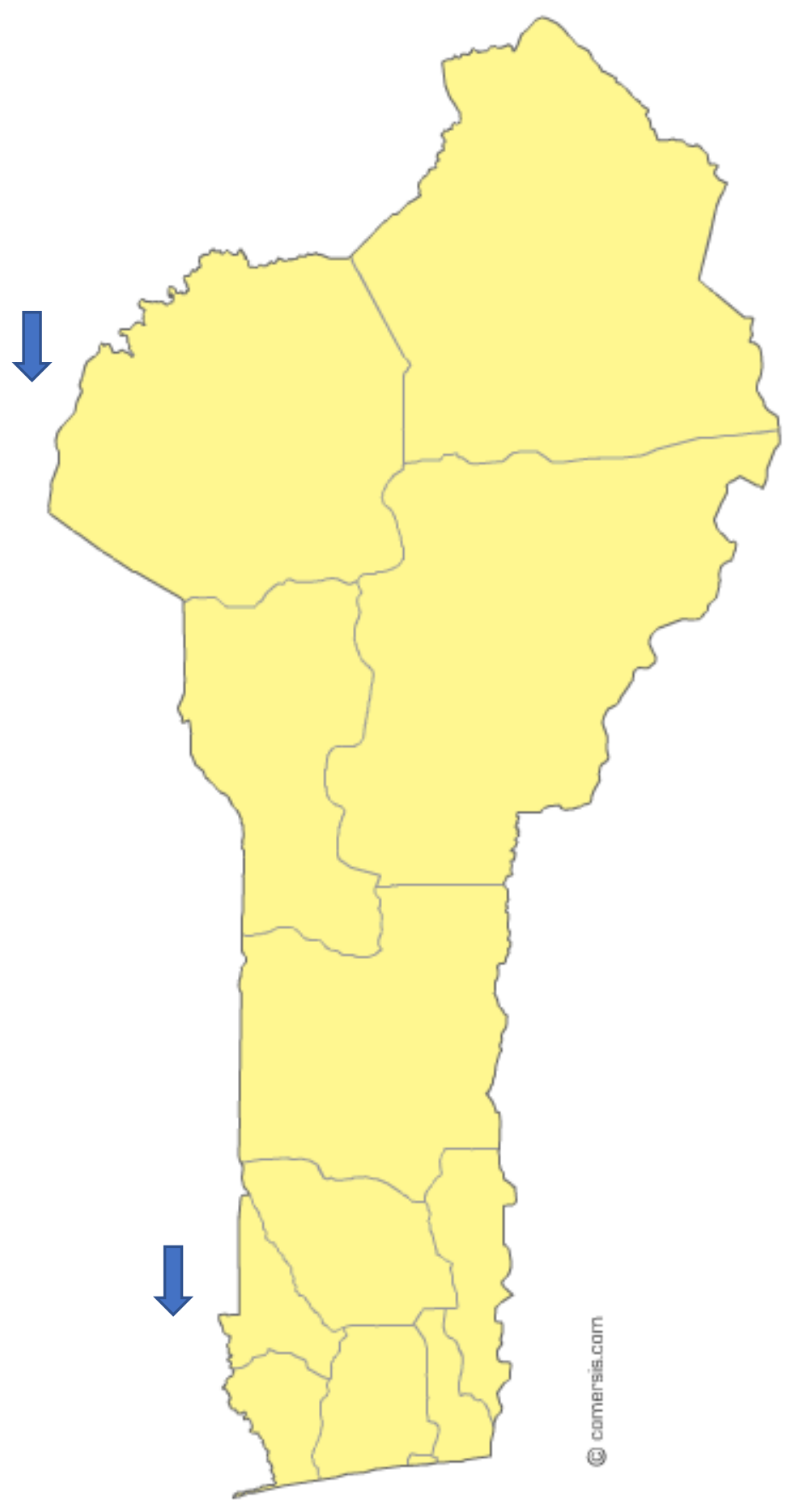

Figure 1 : Carte du Bénin illustrant les départements de l'Atlantique au Sud et de l'Atacora (au Nord). 


\section{RESULTATS}

Elle a concerné 80 phytothérapeutes, herboristes et personnes ressources des départements de l'Atlantique et de l'Atacora.

\section{Profil des Enquêtés \\ Répartition par sexe des enquêtés}

Notre échantillon était composé de 11 femmes et de 69 hommes. Le sexe ratio de 6,27 en faveur du sexe masculin.

\section{Classe socioprofessionnelle}

Notre étude s'est déroulée auprès de 60 tradithérapeutes, 11 personnes ressources et 9 revendeuses de plantes.

\section{Plantes utilisées contre l'infertilité masculine dans le département de l'Atlantique et de l'Atacora}

Ainsi, l'étude a permis de recenser 109 espèces dans les recettes de traitement traditionnel de l'infertilité masculine dans les deux départements. Ces espèces appartiennent à 101 genres et sont réparties en 54 familles parmi lesquelles les plus fréquentes sont représentées dans le Tableau 1, avec leurs parties utilisées et leurs modes d'utilisation. Ce tableau répertorie les espèces les plus fréquentes utilisées pour le traitement de l'infertilité masculine dans les départements de l'Atlantique et de l'Atacora.

\section{Parties utilisées et modes de préparation des recettes}

\section{Parties utilisées}

Pour la préparation des recettes, différentes parties de la plante sont utilisées (feuille, graine, écorce, racine, rhizome /bulbe, fruit) ou même la plante entière.
Les feuilles sont majoritairement utilisées $(35,77 \%)$ suivies des racines $(29,93 \%)$ tandis que les autres parties (fruit, écorce, graines, plante entière, tige feuillée...) sont moins utilisées dans la préparation des recettes (Figure 2).

\section{Modes de préparation des recettes}

La Figure 3 montre le mode de préparation des recettes. La décoction est le mode de préparation majoritairement utilisée soit $33,55 \%$ suivie de la macération $27,63 \%$ et de la poudre $25,66 \%$. La trituration et l'infusion sont moins employées à des fréquences respectives de $11,84 \%$ et $1,32 \%$.

Identification et sélection des plantes les plus significatives

Selon les calculs des indices cités précédemment, les espèces les plus citées sont: Garcinia kola, Citrus aurantifolia, Annona senegalensis, Musa chinensis, Ceropegia deightonii, Carissa spinarum, Manihot esculenta, Rourea coccinea, Cassytha filiformis, Acridocarpus smeathmannii, Pachycarpus lineolatus, Cyperus esculentus, Jatropha gossypiifolia (Tableau 2).

Niveau de connaissance et d'utilisation des espèces à grande variance de citation et leur répartition parmi les enquêtés

Les résultats présentés dans le Tableau 3 montrent que Citrus aurantifolia, Annona senegalensis, Borassus aethiopum, Adansonia digitata ont les indices de diversité (IDmoy) les plus élevés. Ces espèces sont donc bien connues par les enquêtés. Leur indice d'équitabilité (IEmoy) est égal à 1 . Le niveau de connaissance de ces espèces par les enquêtés est réparti alors de manière homogène.

Tableau 1 : Répertoire des espèces les plus fréquentes utilisées pour le traitement de l'infertilité masculine dans les départements de l'Atlantique et de l'Atacora.

\begin{tabular}{llll}
\hline Familles & Espèces & Parties utilisées & Modes d'utilisations \\
\hline Apocynaceae & Carissa spinarum & Feuille, racine & $\begin{array}{l}\text { Décoction, } \\
\text { macération }\end{array}$ \\
\cline { 2 - 4 } & Catharanthus roseus & Feuille & Décoction \\
\cline { 2 - 4 } & Picralina nitida & Ecorce & Macération \\
\cline { 2 - 4 } & Rauvolfia vomitoria & Racine & Macération \\
\cline { 2 - 4 } & Voacanga africana & Racine & Macération \\
\hline
\end{tabular}




\begin{tabular}{|c|c|c|c|}
\hline \multirow[t]{5}{*}{ Annonaceae } & Annona muricata & Feuille, racine & $\begin{array}{l}\text { Trituration, } \\
\text { macération }\end{array}$ \\
\hline & Annona senegalensis & Feuille, racine & $\begin{array}{l}\text { Poudre, macération, } \\
\text { décoction, trituration }\end{array}$ \\
\hline & Monodora myristica & Graines & Macération \\
\hline & Uvaria chamae & Feuille & Décoction \\
\hline & Xylopia aramatica & Grains & Macération \\
\hline \multirow[t]{4}{*}{ Asclepiadaceae } & Calotropis procera & Racine & Poudre \\
\hline & Ceropegia deightonii & Feuille, racine & Macération \\
\hline & Pachycarpus lineolatus & Racine & Macération \\
\hline & Periploca nigrescens & Feuilles & Trituration \\
\hline \multirow[t]{4}{*}{ Caesalpiniaceae } & Burkea africana & Racine & Décoction \\
\hline & $\begin{array}{l}\text { Caesalpinia } \\
\text { benthamianum }\end{array}$ & Plante entière & Décoction \\
\hline & Caesalpinia bonduc & Racines & $\begin{array}{l}\text { Décoction, } \\
\text { macération }\end{array}$ \\
\hline & Caesalpinia pulcherrima & Ecorce, racine, feuille & Décoction \\
\hline \multirow[t]{10}{*}{ Euphorbiaceae } & Bridelia ferruginea & Feuille, écorce & Poudre, décoction \\
\hline & Croton lobatus & Feuille & Décoction \\
\hline & Euphorbia deightonii & Racine & Décoction \\
\hline & Flueggea virosa & $\begin{array}{l}\text { Racine, plante entière, } \\
\text { feuille }\end{array}$ & Décoction, poudre \\
\hline & Hymenocardia neudelotii & Feuille, racine & $\begin{array}{l}\text { Décoction, } \\
\text { macération }\end{array}$ \\
\hline & Jatropha curcas & Feuille, latex, tige & $\begin{array}{l}\text { Trituration, } \\
\text { macération }\end{array}$ \\
\hline & Jatropha gossypiifolia & Feuille, tige & Décoction, infusion \\
\hline & Manihot esculenta & Tubercule, feuille & Poudre, trituration \\
\hline & Ricinus communis & Fruit & Poudre \\
\hline & Sarcocephalus latifolius & Racine & Décoction \\
\hline \multirow[t]{4}{*}{ Fabaceae } & Aganope stuhlmannii & Racine & Décoction \\
\hline & Desmodium velutium & Racine, feuille & Poudre, macération \\
\hline & Pericopsis laxiflora & Racine & Décoction \\
\hline & Philenoptera cyanescens & Feuille & Décoction \\
\hline \multirow[t]{4}{*}{ Mimosaceae } & Copaifera salicunda & Fruit & Macération \\
\hline & Parkia biglobosa & Feuille & Poudre \\
\hline & Prosopis africana & Ecorce & Poudre \\
\hline & Tetrapleura tetraptera & Fruit & Macération \\
\hline \multirow[t]{5}{*}{ Poaceae } & Cenchrus biflorus & Plante entière & Décoction, poudre \\
\hline & Imperata cylindrica & Racine, rhizome & Macération, décoction \\
\hline & Panicum maximum & Feuille & Décoction \\
\hline & Sorghum bicolor & Grain & Poudre, macération \\
\hline & Zea mays & Grain & Macération \\
\hline
\end{tabular}




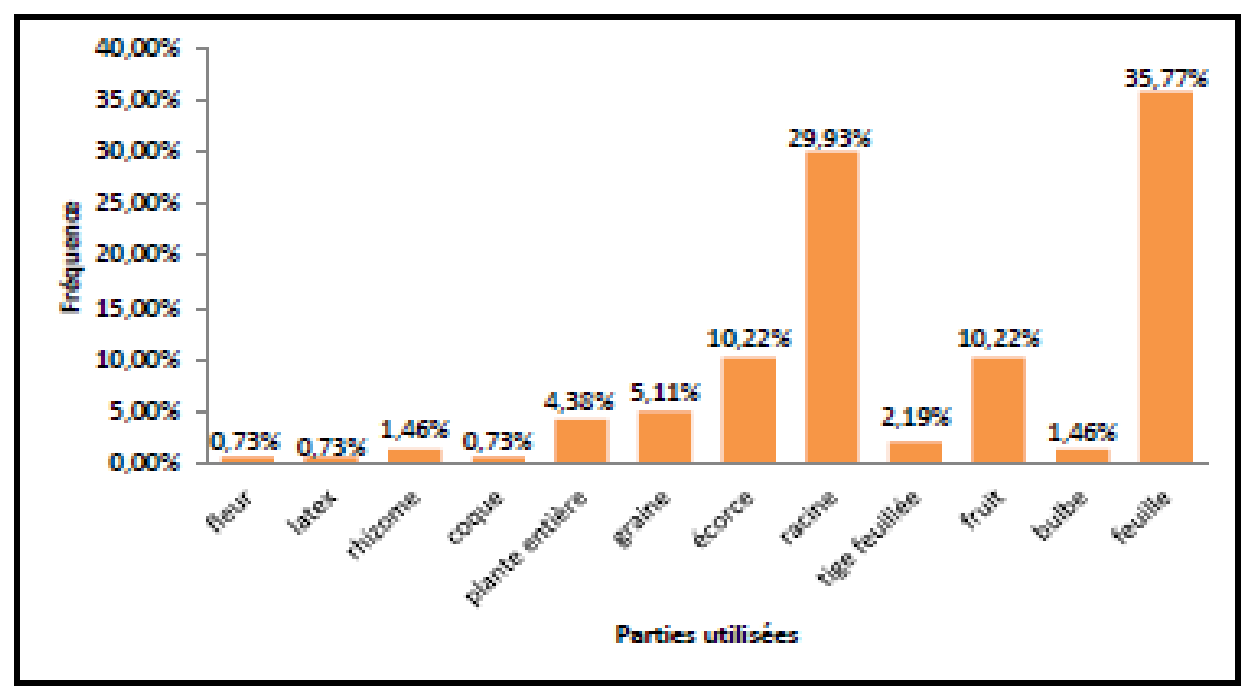

Figure 2 : Partie des plantes utilisées.

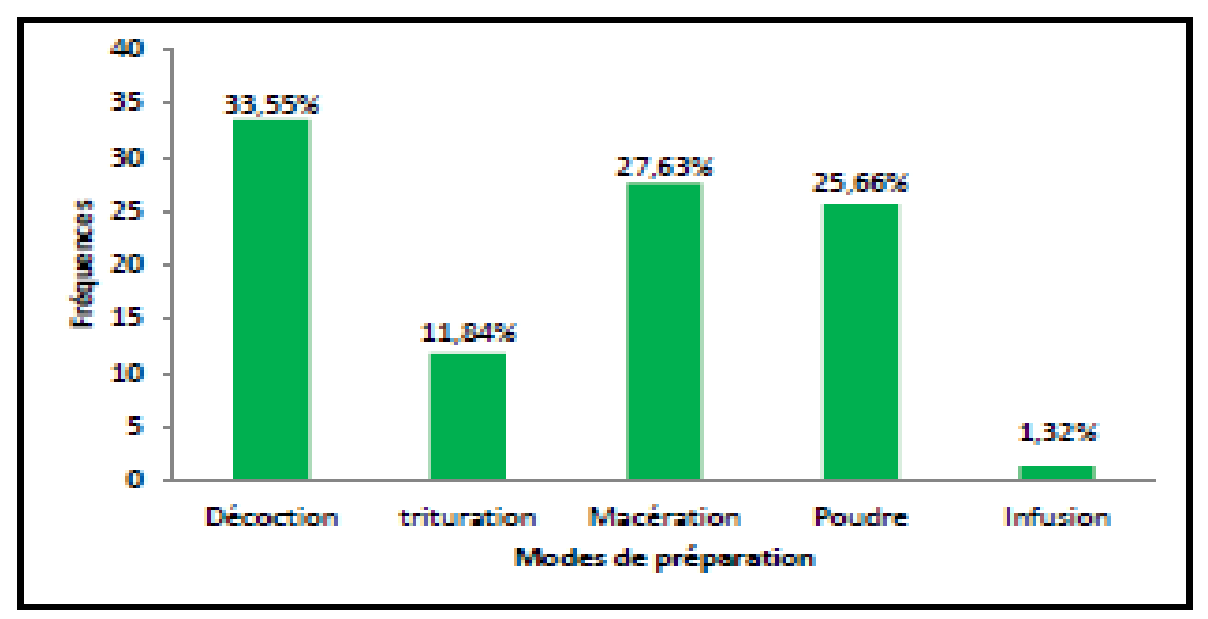

Figure 3 : Mode de préparation des recettes.

Tableau 2 : Espèces les plus citées et fréquence de citation.

\begin{tabular}{lll}
\hline Familles & Espèces & Fréquence de citation en \% \\
\hline Clusiaceae & Garcinia kola & 28,75 \\
Rutaceae & Citrus aurantifolia & 18,75 \\
Annonaceae & Annona senegalensis & 16,25 \\
Musaceae & Musa chinensis & 15 \\
Asclepiadaceae & Ceropegia deightonii & 15 \\
Apocynaceae & Carissa spinarum & 13,75 \\
Euphorbiaceae & Manihot esculenta & 12,5 \\
Connaraceae & Rourea coccinea & 11,25
\end{tabular}




$\begin{array}{lll}\text { Lauraceae } & \text { Cassytha filiformis } & 10 \\ \text { Malpighiaceae } & \begin{array}{l}\text { Acridocarpus } \\ \text { smeathmannii }\end{array} & 8,75 \\ \text { Asclepiadaceae } & \text { Pachycarpus lineolatus } & 7,5 \\ \text { Cyperaceae } & \text { Cyperus esculentus } & 7,5 \\ \text { Euphorbiaceae } & \text { Jatropha gossypiifolia } & 7,5 \\ \text { Annonaceae } & \text { Monodora myristica } & 6,25 \\ \text { Arecaceae } & \text { Borassus aethiopum } & 6,25 \\ \text { Bombacaceae } & \text { Adansonia digitata } & 6,25 \\ \text { Polygalaceae } & \text { Carpolobia lutea } & 6,25 \\ \text { Rutaceae } & \text { Fagara xanthoxyloides } & 6,25\end{array}$

\section{DISCUSSION}

\section{Profil des enquêtés : Sexe et classe socioprofessionnelle}

Au cours de notre étude, nous avons interviewé 60 tradithérapeutes dont 88,33\% étaient des hommes et $11,67 \%$ de femmes. Une prédominance masculine a donc été notée. Nos résultats sont similaires à ceux de Reddy et al. (2009) qui lors d'une enquête ethnobotanique sur les connaissances traditionnelles à propos des plantes médicinales ont trouvé une prédominance masculine avec $78,57 \%$ d'hommes. Par contre, tous les vendeurs de plantes sont de sexe de féminin. Cette féminisation de l'échantillon est due au fait que seules les vendeuses de plantes médicinales ont été questionnées. De plus, au Bénin, il s'agit d'une activité exercée presque exclusivement par les femmes. Nos résultats sont proches de ceux de Muanya et al. (2008) à Lagos au Nigéria, qui ont rapporté une prédominance féminine de $88,9 \%$ au cours d'une enquête sur l'activité aphrodisiaque de quelques espèces de plantes médicinales.

Si la vente des plantes médicinales est une activité féminine, les tradipraticiens et les personnes ressources de notre enquête étaient en majorité des hommes. Nos résultats sont semblables à ceux de Sangaré et al., au Bénin qui ont noté que $80 \%$ d'hommes contre $20 \%$ de femmes utilisaient les plantes médicinales
(Sangaré, 2012). Cela fait évoquer que ce sont les hommes qui utilisent beaucoup plus les plantes médicinales pour le traitement des maladies alors que, les femmes les revendent dans les marchés.

Par contre, ils sont différents de ceux de El Hilah et al. (2016), au Maroc, qui ont trouvé une implication majoritaire des femmes à la fréquence de $65.60 \%$ contre $34.40 \%$ d'hommes. La plupart de nos enquêtés (75\%) étaient des tradithérapeutes en majorité de sexe masculin. En effet, les connaissances traditionnelles sont transmises d'une part de génération en génération, de père en fils et d'autre part, acquises par apprentissage d'un guide aux hommes le plus souvent considérés comme gardien de la tradition, par opposition aux femmes qui étaient à tort considérées comme incapables de garder les secrets.

\section{Renseignements sur les plantes}

Notre étude a permis de répertorier 109 espèces utilisées dans le traitement de l'infertilité masculine. Cette multiplicité de traitement démontre de la richesse thérapeutique de la médecine traditionnelle africaine et celle béninoise en particulier. Les familles les plus représentées sont les Euphorbiaceae, les Caesalpiniaceae, les Poaceae, les Annonaceae, les Apocynaceae, les Asclepiadaceae, les Fabaceae et les 
Mimosaceae. Dans une étude effectuée au Cameroun, Jiofack et al. (2009) ont retrouvé parmi les familles les plus représentées, les Mimosaceae et les Apocynaceae. De même, Nduche et al. (2015) ont noté que les familles de plantes les plus utilisées dans l'Etat de Ebonyi au Nigéria sont : les Euphorbiaceae, les Fabaceae, les Apocynaceae et les Annonaceae. Erhabor et al. (2013) ont quant à eux relevé les familles suivantes: Arecaceae, Cucurbitaceae, Liliaceae, Musaceae et Zingiberaceae. Sur les 109 espèces, 87 ont été déjà rapportées par des études antérieures au Nigeria et au Cameroun, ce qui témoigne de l'utilisation réelle de ces plantes dans le traitement de l'infertilité masculine dans plusieurs pays (Kaundal, 2016 ; Olowokudejo, 2008 ; Telefo, 2011).

\section{Parties utilisées}

Dans la préparation des recettes, toutes les parties de plantes sont utilisées mais, ce sont les feuilles qui le sont majoritairement à $35,77 \%$. Plusieurs études ont également noté ces résultats. En effet, nos résultats concordent avec ceux de Catarino et al. (2016) en Guinée et Nduche et al. (2015), qui ont trouvé respectivement que $34.9 \%$ et $37,5 \%$ des feuilles étaient utilisées. De même, Balamurugan et al. (2017) en Inde ainsi que Magassouba et al. (2007) en Guinée ont relevé une forte utilisation des feuilles lors de leurs études respectives : $32 \%$ et $40,8 \%$. Au Nigéria, Adebayo et al. (2011), ainsi que Fokou et al. (2015), ont noté que ce sont les feuilles qui étaient le plus utilisées dans les recettes traditionnelles respectivement à $44,26 \%$ et 53\%. Allabi et al. (2011) au Bénin ont trouvé que les feuilles sont utilisées dans $55 \%$ des cas. L'utilisation des feuilles présente peu de dangers pour la plante. L'aisance et la rapidité de la récolte des feuilles pourraient expliquer leur utilisation accrue.

Hormis les feuilles, les parties de plantes qui sont le plus utilisées sont les racines puis l'écorce et le fruit, respectivement à 29,9\% et $10,22 \%$. Allabi et al. (2011), au Bénin ont trouvé également une faible utilisation des racines et des écorces du tronc respectivement à une fréquence de $22 \%$ et $11 \%$. Nos résultats concordent aussi avec ceux de Balamurugan et al. (2017) en Inde qui ont rapporté une utilisation des racines à $20 \%$ et celle des écorces du tronc à $12 \%$. Cependant, le prélèvement de la racine ou de l'écorce du tronc de façon anarchique pourrait être responsable de la disparition de certaines espèces.

\section{Modes de préparation et voie d'administration}

La méthode de préparation la plus utilisée est la décoction $(33,55 \%)$. Fokou et al. (2015) au Nigéria et Soladoye et al. (2010) ont également noté que la décoction était la méthode la plus utilisée. Aussi, il faut noter que toutes les recettes sont utilisées par voie orale dans la plupart de la pharmacopée africaine (Ajibesin, 2008).

\section{Conclusion}

Les résultats obtenus ont permis de montrer la richesse de la flore béninoise en ressources pour la prise en charge de l'infertilité masculine.

\section{CONFLIT D'INTERETS}

Il n'existe aucun conflit d'intérêts entre les auteurs de ce manuscrit.

\section{CONTRIBUTIONS DES AUTEURS}

Les auteurs SA et CK ont contribué de façon équitable à ce travail. MA, BA ont assuré l'analyse statistique du travail. MAD s'est occupé de la coordination du plateau technique d'analyse des échantillons. BAW, AL, MPA, RBD ont assuré la coordination scientifique de ce travail de recherche académique. Tous les auteurs ont participé à la rédaction de l'article et ont donné leur correction.

\section{REMERCIEMENTS}

Nous remercions également les habitants de Kika et de Parakou qui ont participé à cette étude.

\section{REFERENCES}

Adebayo JO, Krettli AU. 2011. Potential antimalarials from Nigerian plants: a review. J Ethnopharmacol, 133: 289-302. DOI: $10.1016 /$ j.jep.2010.11.024. 
Agarwal A, Mulgund A, Hamada A, Chyatte MR. 2015. A unique view on male infertility around the globe. Reprod. Biol. Endocrinol, 13(1): $37 . \quad$ DOI: 10.1186/s12958-015-0032-1.

Ajibesin K, Ekpoa BA, Danladi N. Bala A, Etienne E. Essien B, Saburi A. Adesanya C. 2008. Ethnobotanical survey of Akwa Ibom State of Nigeria Kola. $J$ Ethnopharmacol, 115: 387-408. DOI: 10.1016/j.jep.2007.10.021.

Akour A, Kasabri V, Fatma UA, Bulatova N. 2015. The use of medicinal herbs in gynecological and pregnancy-related disorders by Jordanian women: a review of folkloric practice vs. evidence-based pharmacology. Pharmaceutical Biology, 54: $9, \quad 1901-1918, \quad$ DOI: 10.3109/13880209.2015.1113994

Allabi AC, Busiac K, Ekanmiana V, Bakiono F. 2011. The use of medicinal plants in self-care in the Agonlin region of Benin. $J$ Ethnopharmacol, 133: 234-243. DOI: 10.1016/j.jep.2010.09.028.

Balamurugan S, Vijayakumar S, Prabhu S, Yabesh JE. 2017. Traditional plants used for the treatment of gynaecological disorders in Vedaranyam taluk, South Indian: an ethnomedicinal survey. $J$ Tradit Complement Med. 1: 1-16. DOI: 10.1016/j.jtcme.2017.06.009.

Brzakowski M, Lourdel E, Cabry R, Oliéric MF, Claeys C, Devaux A et al. 2009. Epidémiologie du couple infertile. Journal de Gynéco-obstétrique et Biologie de la Reproduction, 38 (1) : 9$18 . \quad$ DOI: $\quad 10.1016 / \mathrm{S} 0368-$ 2315(09)70226-1.

Byg A, Baslev H. 2001. Diversity and use of palms in Zahamena, eastern Madagascar. Biodivers Conserv., 10: 951-70. DOI : $10.1023 / \mathrm{A}: 1016640713643$.

Catarino L, Havik P, Indjai B, Romeiras M. 2016. Ecological data in support of an analysis of Guinea-Bissau's medicinal flora. Data in Brief, 7: 1078-1097. DOI: https://doi.org/10.1016/j.dib.2016.03.077

Djego J, Djego-djossou S, Cakpo Y, Agnani P, Sinsin B. 2011. Evaluation du potentiel ethnobotanique des populations rurales au
Sud et au centre du Bénin. Int. J. Biol. Chem. Sci., 5(4): 1432-1447. DOI: $10.4314 /$ ijbcs.v5i4.10.

Erhabor JO, Idu M, Udo FO. 2013. Ethnomedicinal Survey of Medicinal Plants Used in the Treatment of Male Infertilty among the IFA Nkari People of Ini Local Government Area of Akwa Ibom State, Nigeria. Res J Recent Sci., 2: 5-11.

El hilah F, Ben Akka F, Bengueddour R, Rochdi A, Zidane L. 2016. Etude ethnobotanique des plantes médicinales utilisées dans le traitement des affections dermatologiques dans le plateau central marocain. J. Appl. Biosci., 98: 9252 9260. DOI: $10.4314 /$ jab.v98i1.2

Fokou P, Nyarko A, Appiah-opong R, Yamthe L, Addo P, Asante I, Fekam Boyom F. 2015. Ethnopharmacological reports on anti-Buruli ulcer medicinal plants in three West African countries. $J$ Ethnopharmacol., 172: 297-311. DOI: 10.1016/j.jep.2015.06.024.

Jiofack T, Ayissi I, Fokunang C, Guedje N, Kemeuze V. 2009. Ethnobotany and phytomedicine of the upper Nyong valley forest in Cameroon. Afr $J$ Pharm Pharmacol., 3(4): 144-150.

Jimoh AG, Olawuyi TS, Omotoso GO, Oyewopo AO, Dare JK. 2012. Semen parameters and hormon profile of men investigated for infertility at midland fertility center. J B App Sci., 8:16-19.

Kaundal A, Rana R, DeviM. 2016. Infertility treatment using herbal drugs: a review. Eur J Pharm Med Res., 3(7):194-201. DOI: https://doi.org/10.1186/s40545019-0200-3.

Muanya CA, Odukoya OA. 2008. Lipid Peroxydation as Index of Activity in Aphrodisiac Herbs. Plant Sci J., 3(1): 9298. DOI: $10.3923 /$ jps.2008.92.98.

Magassouba FB, Diallo A, Kouyat M, Mara F, Mara O, Bangoura O, Camara A, Traoré S, Diallo AK, Zaoro M, Lamah K, Diallo S, Camara G, Traoré S, Kéita A, Camara MK, Barry R, Kéita S, Oularé K, Barry MS, Donzo 
M, Camara K, Toté K, Vanden Berghe D, Totté J, Pieters L, Vlietinck AJ, Baldé AM. 2007. Ethnobotanical survey and antibacterial activity of some plants used in Guinean traditional medicine. J Ethnopharmacol., 114: 4453. DOI: 10.1016/j.jep.2007.07.009.

Monteiro JM, Albuquerque UP, Lins Neto EMF, AraújoEL, AmorimELC. 2006. Use patterns and knowledge of medicinal species among tworural communities in Brazil's semi-arid northeastern region. $J$ Ethnopharmacol., 105:173-86. DOI: 10.1016/j.jep.2005.10.016.

Nduche MU, Omosun G, Okwulehie IC. 2015. Ethnobotanical Survey of Plants Used as Remedy for Fertility Conditions in Ebonyi State of Nigeria. Sch Acad J Biosci., 3 (2B): 214-221.

Olowokudejo JD, Kadiri AB, Travih VA. 2008. An Ethnobotanical Survey of Herbal Markets and Medicinal Plants in Lagos State of Nigeria. Ethnobotanical Leaflets, 12: 851-865. Disponible sur : https://opensiuc.lib.siu.edu/ebl/vol2008/i ss 1/116.

Reddy CS, Reddy KN, Murthy EN, Raju VS. 2009. Traditional medicinal plants in Seshachalam hills, Andhra Pradesh, India. J Med Plant Res., 3(5): 408-412.

Sangaré M, Sina H, Dougnon J, Bayala B, Ategbo J, Dramane KL. 2012. Etude ethnobotanique des plantes hépatotropes et de l'usage traditionnel de Gomphrena celosioides Mart. (Amaranthaceae) au
Bénin. Int. J. Biol. Chem. Sci., 6(6): 50085021. DOI: 10.4314/ijbcs.v6i6.20.

Sifroi JP, Le Bourhis C, Krausz C, Dadoune JP, Fellous M. 2000. Infertilité masculine : des anomalies moléculaires aux possibilités thérapeutiques. Médecine/Sciences, 15: 307-315. DOI : 10.1016/j.gofs.2018.11.004

Soladoye MO, Adetayo MO, Chukwuma EC, Adetunji AN. 2010. Ethnobotanical survey of plants used in the treatment of haemorroids in South-Western Nigeria. Ann Biol Res., 1(4):1-15. www.scholarsresearchlibrary.com

Telefo PB, Lemfack MC, Bayala B, Lienou LL, Goka CS, Yemele MD. 2011. Ethnopharmacological survey of plants used for the treatment of female infertility in Baham, Cameroon. $J$ Ethnopharmacology, 136: 178-187. DOI: 10.1016/j.jep.2011.04.036.

World Health Organization. 2010. WHO Laboratory Manual for the Examination and Processing of Human Semen (5th edition). Cambridge University Press: Cambridge, UK.

Zohoun TH, Flenon J. 1997. La Médecine traditionnelle et la pharmacopée africaine peuvent-elles constituer une alternative de soins face aux coûts prohibitifs actuels de la Médecine moderne ? In Pharmacopée et Médecine Traditionnelle Africaine. Les Presses de l'U.B. : Lomé ; 3-15. 